\title{
VALIDACIÓN DE LA ESCALA DE CREENCIAS DE EFICACIA EN LA ENSEÑANZA DE LA MATEMÁTICA (ECEEM) Y CARACTERIZACIÓN DE LAS CREENCIAS DE ESTUDIANTES DE PEDAGOGÍA BÁSICA ${ }^{1}$
}

\author{
Marco Verdugo ${ }^{2}$ \\ Rodrigo Asún ${ }^{3}$ \\ Salomé Martínez ${ }^{4}$
}

RESUMEN

Tomando en cuenta la relevancia de que los estudiantes de Pedagogía tengan creencias positivas acerca de su futura eficacia como docentes y la ausencia de instrumentos validados en la población chilena que midan dicho constructo, en esta investigación se realizó la validación convergente y factorial de la Escala de Creencias de Eficacia en la Enseñanza de la Matemática (ECEEM), adaptación al español de la escala Mathematics Teaching Efficacy Belief Instrument (MTEBI), validada en Estados Unidos en el año 2000. La muestra estuvo conformada por 945 estudiantes de Pedagogía en Educación Básica de 14 universidades en cinco regiones, correspondientes a las zonas norte, centro y sur de Chile. Los resultados indicaron que ECEEM presentó buen nivel de discriminación, un ajuste adecuado a la estructura teórica propuesta, alta fiabilidad y una correlación significativa con las variables esperadas, por lo que constituye un instrumento adecuado para medir las creencias de eficacia en la enseñanza de la matemática de futuros docentes de Pedagogía Básica. En términos descriptivos, los futuros docentes mostraron creencias de eficacia positivas, pero no totalmente satisfactorias, pues existe un grupo importante que duda de las posibilidades de producir aprendizajes significativos en sus estudiantes.

Palabras clave: creencias de eficacia en la enseñanza de la matemática, estudiantes de Pedagogía en Educación Básica, formación inicial docente, medición, validación.

Esta investigación fue financiada por los proyectos Fondef IT 13I10005, PIA-Conicyt Proyecto BF0003 y Proyecto Basal CMM U. de Chile and UMI2807 CNRS.

2 Investigador independiente, Santiago, Chile. Contacto: marco.verdugoa@gmail.com

3 Facultad de Ciencias Sociales, Universidad de Chile, Santiago, Chile. Contacto: rasun@uchile.cl

4 Facultad de Ciencias Físicas y Matemáticas, Universidad de Chile, Santiago, Chile. Contacto: samartin@dim.uchile.cl 


\title{
VALIDATION OF AN ADAPTATION IN SPANISH OF THE MATHEMATICS TEACHING EFFICACY BELIEF INSTRUMENT (MTEBI) AND CHARACTERIZATION OF PRESERVICE PRIMARY EDUCATION TEACHERS' BELIEFS
}

\begin{abstract}
Considering the relevance of preservice teachers possessing positive beliefs of their future efficacy as teachers and the absence of validated instruments to measure this construct in Chile, this research generated a convergent and factorial validation of the Escala de Creencias de Eficacia en la Enseñanza de la Matemática (ECEEM), the adapted version in Spanish of the Mathematics Teaching Efficacy Beliefs Instrument (MTEBI), validated in the United States, in 2000. The sample was comprised of 945 preservice elementary school teachers studying in 14 universities from 5 regions corresponding to northern, central and southern Chile. The results indicate that ECEEM showed a good level of discrimination, an adequate adjustment to the proposed theoretical structure, a high reliability and a significant correlation with the expected variables. Thus, it serves as a reliable instrument to measure the mathematics teaching efficacy beliefs of preservice primary education teachers. In descriptive terms, the preservice teachers showed positive efficacy beliefs, though not completely satisfactory, since a significant group doubt the possibility of producing meaningful learning opportunities for their students.
\end{abstract}

Keywords: initial teacher training, math teaching efficacy beliefs, measurement, , validation.

\section{Introducción}

La investigación actual en educación ha dejado cada vez más claro que el desempeño de los docentes no depende solamente de su nivel de conocimientos, sino también de sus creencias, actitudes y percepciones respecto del aprendizaje y de su rol como pedagogos, de manera que creencias positivas o negativas respecto de su capacidad de enseñar o incidir en el futuro de sus estudiantes afecta su rendimiento como educadores (Hidalgo, Maroto, Ortega y Palacios, 2014; Hidalgo, Maroto y Palacios, 2000; 2004; Hoffman, 2010; Olaz, 2001; Wenta, 2000). En vista de ello, es posible suponer que un sistema educativo que no sea capaz de mejorar las expectativas de los futuros docentes respecto del rol que puedan cumplir en el proceso de aprendizaje de sus estudiantes y de la capacidad que posean de enseñar y conseguir buenos resultados, podrá mejorar solo marginalmente su calidad. Lamentablemente, en Chile no se dispone de instrumentos validados que midan este tipo de variables subjetivas, por lo que ni las autoridades ni las universidades 
pueden hacer seguimiento y diagnóstico de su estado y evolución. Se requiere, entonces, desarrollar e implementar instrumentos que midan creencias, actitudes y percepciones críticas para el futuro desempeño de los educadores.

Se ha evidenciado que, dentro del conjunto de creencias, actitudes y percepciones de los futuros profesores, las creencias acerca de su eficacia en la enseñanza de la matemática son relevantes, pues se relacionan favorablemente con su desempeño educativo (Moseley \& Utley, 2006); con su calidad de vida en general (Velásquez, 2012); con una mejor adaptación a innovaciones educativas (De Mesquita $\&$ Drake, 1994; Ghaith \& Yaghi, 1997; Guskey \& Passaro, 1994); con una mayor flexibilidad para emplear distintos enfoques al desarrollar los contenidos; con la incorporación de nuevas ideas y destrezas; y con la disposición a cambiar los métodos de enseñanza (Czerniak \& Lumpe, 1996; Ghaith \& Yaghi, 1997).

Producto de la importancia de este constructo para el desempeño y las actitudes docentes, no es de extrañar que se hayan desarrollado instrumentos estadísticos para medirlo en estudiantes de Pedagogía y docentes de cursos de matemática o ciencia, como es el caso de las escalas Mathematics Teaching Efficacy Belief Instrument (MTEBI) (Enochs, Smith \& Huinker, 2000) y Science Teaching Efficacy Belief Instrument-Preservice (STEBI-B) (Enochs \& Riggs, 1990; Enochs et al., 2000), y que estos instrumentos se hayan empleado para evaluar el impacto de estas variables en el aprendizaje en la literatura científica internacional (ver por ejemplo: Alkhateeb, 2004; Bleicher, 2004; Cetinkaya \& Erbas, 2011; Moseley \& Utley, 2006; Swars, 2005). Sin embargo, estas escalas han sido diseñadas y validadas en idiomas distintos al español y en contextos diferentes al latinoamericano (ver por ejemplo: Alkhateeb, 2004; Cetinkaya \& Erbas, 2011; Enochs et al., 2000; Moseley \& Utley, 2006), por lo que para ser utilizadas en América Latina requieren ser sometidas previamente a un proceso de validación de sus versiones en español.

Frente a esto, la presente investigación se propuso llenar dicho vacío a través de la validación convergente y factorial de la 
Escala de Creencias de Eficacia en la Enseñanza de la Matemática (ECEEM) — que es una adaptación al español de la escala MTEBI-, con el objetivo de aportar un instrumento que pueda ser empleado en estudios académicos y evaluaciones aplicadas en países de habla hispana. Además, se realizó una primera caracterización de los futuros docentes de Pedagogía Básica en Chile, respecto de las creencias que poseen acerca de los aprendizajes matemáticos que puede generar su desempeño profesional en sus futuros estudiantes de educación básica.

Lo anterior puede sintetizarse en los siguientes objetivos de investigación:

Objetivo general: determinar el grado de validez y fiabilidad de la Escala de Creencias de Eficacia en la Enseñanza de la Matemática (ECEEM) y caracterizar a los estudiantes universitarios de Pedagogía Básica en Chile, según sus creencias sobre los aprendizajes matemáticos que puede generar su futuro desempeño profesional.

Objetivos específicos:

- determinar el nivel de fiabilidad por consistencia interna de la escala ECEEM;

- determinar el nivel de validez por estructura interna y convergencia de la escala ECEEM;

- describir las creencias de eficacia en la enseñanza de la matemática que presentan los estudiantes universitarios de Pedagogía Básica en Chile.

\section{Antecedentes conceptuales}

\subsection{La relación entre las creencias y el rendimiento matemático}

El rendimiento matemático emerge de la relación entre variables de atributo (como el conocimiento y las capacidades) y el dominio afectivo de los estudiantes (emociones, creencias, actitudes) (Hidalgo et al., 2014). Representaciones tales como el autoconcepto matemático, las creencias matemáticas, las creencias acerca del 
profesor o la percepción de dificultad (Gómez, 1997; Hidalgo et al., 2000; 2004) condicionan la percepción y modo de actuar de los sujetos, de manera que las personas tienden a intervenir activamente en contextos donde se sienten eficaces, pero evitan situaciones donde se piensan ineficaces (Olaz, 2001), posibilitando u obstaculizando la adquisición de habilidades y destrezas que entreguen esos contextos.

Por otro lado, el rendimiento también afecta el tipo de representaciones de los sujetos sobre sí mismos (Velásquez, 2012), por lo que las dificultades aparejadas al desarrollo de un pensamiento matemático superior (Hidalgo et al., 2004) y la vivencia subjetiva de dichas dificultades condicionan el tipo de creencias que desarrolla el sujeto acerca de su eficacia en matemática. En consecuencia, la interpretación que el profesor haga de los resultados de sus acciones afectará a su desempeño posterior como docente y viceversa (Czerniak, 1990; Hoffman, 2010; Wenta, 2000). No obstante, no todo el dominio afectivo de las personas influye en igual magnitud en los desempeños docentes. Como se verá a continuación, las creencias de eficacia en la enseñanza ocupan un lugar destacado.

\subsection{Creencias de eficacia en la enseñanza de la}

matemática. La autoeficacia y expectativas de resultado de la enseñanza

El concepto creencias de eficacia en la enseñanza está basado en la teoría de la autoeficacia de Bandura (1997), que tiene como supuesto fundamental que las creencias autorreferentes permiten explicar en gran medida el comportamiento de los sujetos (Blanco, 2010; Olaz, 2001).

Las creencias de eficacia son un tipo de creencias ligadas a ámbitos de funcionamiento diferenciado, dinámicas y aprendidas en contextos específicos (Bandura, 1997). Circunscritas al ámbito de la enseñanza, pueden ser entendidas como los juicios que el maestro posee acerca de las posibilidades de influir en el nivel de aprendizaje de los estudiantes (Guskey \& Passaro, 1994) o, en el caso de los futuros docentes, como las creencias que tienen los estudiantes de Pedagogía respecto de los aprendizajes que puede generar su futuro desempeño profesional. 
150 VALIDACIÓN DE LA ESCALA DE CREENCIAS DE EFICACIA EN LA ENSEÑANZA DE LA MATEMÁTICA (ECEEM) Y CARACTERIZACIÓN DE LAS CREENCIAS DE ESTUDIANTES DE PEDAGOGÍA BÁSICA - M. Verdugo, R. Asún y S. Martínez

Operacionalmente, el constructo creencias de eficacia se constituye a partir de dos dimensiones: la autoeficacia y las expectativas de resultado (Ashton, 1985; Dembo \& Gibson, 1985, 1985; Enochs et al., 2000). Las expectativas de resultado son los juicios sobre los posibles resultados causados por un comportamiento o una acción determinada, independiente de quien la realice (Bandura, $1977 ;$ 1986; 1997). En cambio, la autoeficacia es la percepción acerca de la capacidad de uno mismo para ejecutar con éxito el comportamiento requerido para producir los resultados esperados (Bandura, 1977; 1986; 1997). Es decir, "mientras que la autoeficacia se refiere a las expectativas respecto de nuestras propias capacidades, las expectativas de resultado aluden a las creencias acerca de qué ocurrirá como consecuencia de los esfuerzos comportamentales" (Cupani y Gnavi, 2007, p. 19). Aplicado a la docencia, mientras la autoeficacia docente representa la creencia de un profesor en torno a sus capacidades y habilidades para ser un maestro eficaz o determinante en el aprendizaje de los estudiantes, la expectativa de resultado sería la creencia en la potencialidad de la docencia para afectar en la formación de los estudiantes, independientemente de los factores externos (Swars, 2005).

Las investigaciones respecto de la relación entre las creencias de eficacia y algunas variables significativas para el proceso educativo han evidenciado que aquellos docentes que tienen altas y positivas creencias de eficacia en la enseñanza suelen tener menores niveles de ansiedad matemática (Swars, 2004), tienden a utilizar una mayor variedad de estrategias de enseñanza (Wenta, 2000), y emplean con mayor frecuencia estrategias de enseñanza centradas en el estudiante (Czerniak, 1990) o técnicas de enseñanza complejas (Czerniak \& Lumpe, 1996). Coincidentemente, conductas tales como la persistencia en una tarea, el asumir riesgos y el uso de innovaciones - que implican un mayor rendimiento de los sujetosestán relacionadas con grados mayores de eficacia percibida (Ashton, 1985). A su vez, los docentes que tienen un peor juicio acerca de la eficacia de la enseñanza de la matemática son más propensos a utilizar estrategias dirigidas por el maestro, sin dar mucho espacio para la libertad del estudiante (Czerniak, 1990). 


\subsection{La medición de las creencias de eficacia}

En un primer momento, la idea general de que las creencias de eficacia de un docente son un juicio de su capacidad para lograr resultados deseados en torno al aprendizaje dio origen a un grupo de instrumentos que solo consideraron la dimensión de autoeficacia en su operacionalización (Tschannen-Moran \& Hoy, 2001). Este es el caso de las primeras medidas del constructo creencias de eficacia docente que, basadas en las teorías del aprendizaje social de Rotter (1966), solo preguntaban por las creencias del docente acerca de su capacidad para impactar en el aprendizaje de un estudiante (autoeficacia). Si bien estos esfuerzos no permearon mayormente la investigación científica (Tschannen-Moran \& Hoy, 2001), dieron origen a un instrumento de dos ítems, que luego se dejó de emplear cuando se desarrollaron escalas más sofisticadas (Tschannen-Moran \& Hoy, 2001), que buscaban mejorar la medición y fiabilidad de la escala original (Rose \& Medway, 1981) o incorporar elementos contextuales (Ashton, Buhr \& Crocker, 1984).

Desde los años ochenta, se desarrolló una segunda línea metodológica apegada a los postulados de la teoría social cognitiva de Bandura (Tschannen-Moran \& Hoy, 2001). En ella, diversos estudios ligaron la autoeficacia y las expectativas de resultado con las creencias de eficacia (Cetinkaya \& Erbas, 2011), lo que dio lugar al desarrollo de nuevas escalas, que incorporaron las expectativas de resultado como subescala de las creencias de eficacia (Gibson \& Dembo, 1984). Particularmente, fue la escala Teacher Efficacy Scale (TES) el punto de partida de nuevos instrumentos para medir las creencias de eficacia, a partir de un modelo bifactorial (Cetinkaya \& Erbas, 2011).

Influenciados por los postulados de la teoría de la autoeficacia (Bandura, 1977; 1978; 1984; 1986; 1995; 1997; 1998; 2004; 2006), se asumió que es el factor autoeficacia el que tendría un mayor poder explicativo sobre el comportamiento, en la medida en que los resultados que las personas anticipan dependen, en gran parte, de sus valoraciones sobre cuán bien podrán comportarse en determinadas situaciones. Sin embargo, algunos autores han cuestionado la supuesta relevancia secundaria de las expectativas de 
152 VALIDACIÓN DE LA ESCALA DE CREENCIAS DE EFICACIA EN LA ENSEÑANZA DE LA MATEMÁTICA (ECEEM) Y CARACTERIZACIÓN DE LAS CREENCIAS DE ESTUDIANTES DE PEDAGOGÍA BÁSICA - M. Verdugo, R. Asún y S. Martínez

resultado (ver por ejemplo: Corcoran, 1995; Kirsch, 1995), pues, aun cuando un sujeto crea poseer las habilidades para realizar un comportamiento, es probable que no lo realice si considera que los resultados esperados no lo motivan (Rhodes \& Courneya, 2003) o si observa que el impacto en el rendimiento de los estudiantes no se produce (Dellinger, Bobbett, Olivier \& Ellett, 2008). En consecuencia, algunas veces la aparente influencia de la autoeficacia sobre el comportamiento se debe realmente a la presencia de buenas expectativas de resultado (Corcoran, 1995; Kirsch, 1995), en tanto la presencia de autoeficacia sin buenas expectativas de resultado disminuiría la relación de la primera con otros dominios de comportamiento (Williams, 2010). Asumiendo esta problemática, la escala que se validará en esta investigación -ECEEM- posee una estructura bifactorial, diferenciándose las dimensiones de autoeficacia y expectativas de resultado, por lo que se asume que ambos constructos son significativos para entender el comportamiento, tal como recomienda Williams (2010).

Finalmente, las creencias de eficacia están ligadas a ámbitos de funcionamiento específicos que, en el caso de los docentes, no se comportan de manera uniforme a través de los diferentes tipos de tareas docentes ni ámbitos del conocimiento en que se deban desempeñar (Bandura, 1997), por lo que su medición requiere de instrumentos focalizados en una sola tarea. Una de las primeras escalas que se enfocó específicamente en la tarea de la enseñanza de la ciencia fue la Science Teaching Efficacy Belief Instrument-Preservice (STEBI-B) (Enochs \& Riggs, 1990), para luego ser la base de la escala Mathematics Teaching Efficacy Belief Instrument (MTEBI) (Enochs et al., 2000). Esta última aborda las debilidades de la escala TES (Cetinkaya $\&$ Erbas, 2011), y fue diseñada para un asunto y sujeto específico: la enseñanza de la matemática en estudiantes de Pedagogía (Enochs et al., 2000). Junto con ello, el instrumento mide estas creencias a partir de diversos ítems, sin perder poder explicativo sobre la conducta debido al aumento de la especificidad de la escala, como temía Pajares (1996). 


\subsection{La escala MTEBI: propiedades psicométricas y utilización}

La Escala de Creencias de Eficacia en la Enseñanza de la Matemática (ECEEM) es una adaptación al español de la escala Mathematics Teaching Efficacy Belief Instrument (MTEBI) validada en Estados Unidos por Enochs y colaboradores (2000). A su vez, el MTEBI es una adaptación del Science Teaching Efficacy Belief Instrument (STEBI-B), que fue construida sobre la base de la escala Teacher Efficacy Scale (TES).

Mientras la escala MTEBI pretende medir las creencias de eficacia en la enseñanza de la matemática (Enochs et al., 2000), STEBI-B se enfoca en el ámbito de la ciencia (Enochs \& Riggs, 1990). Como el fraseo de los ítems de MTEBI y STEBI-B es muy similar (en muchas ocasiones solo se cambió el término science por mathematics), y la información disponible acerca de las propiedades psicométricas de MTEBI es aún limitada (Cetinkaya, 2011), es pertinente reportar las propiedades psicométricas y los usos de ambas escalas conjuntamente.

La versión original de la escala STEBI-B constaba de 25 ítems, los que fueron reducidos a 23 luego de que su estructura factorial y fiabilidad fuera analizada (Enochs \& Riggs, 1990). La STEBI-B se posicionó como una herramienta importante para los investigadores de educación científica (Moseley \& Utley, 2006) y fue a partir de esta última versión que surgió MTEBI, también compuesta por 23 ítems (Enochs et al., 2000).

La validación factorial de MTEBI, realizada por Enochs y colaboradores (2000), concluyó que el modelo bifactorial ajustaba con los datos, pero dos ítems presentaron baja correlación con la variable latente, lo que llevó a los investigadores a eliminarlos. Además, algunos ítems tuvieron más relación entre sí que el atribuible al constructo latente, lo que llevó a los autores a introducir en el modelo correlaciones entre los errores que no se recomiendan en la literatura especializada, pues indican que parte de la covariación entre los ítems no se explica por el constructo latente que miden. No obstante lo anterior, MTEBI se constituyó por 21 ítems tipo 
154 VALIDACIÓN DE LA ESCALA DE CREENCIAS DE EFICACIA EN LA ENSEÑANZA DE LA MATEMÁTICA (ECEEM) Y CARACTERIZACIÓN DE LAS CREENCIAS DE ESTUDIANTES DE PEDAGOGÍA BÁSICA - M. Verdugo, R. Asún y S. Martínez

Likert de cinco puntos, distribuidos en dos subescalas que miden la Autoeficacia en la Enseñanza de la Matemática (AEM), con 13 ítems, y las Expectativas de Resultado en la Enseñanza de la Matemática (EREM), con ocho ítems. Además, el análisis de fiabilidad arrojó un alfa de Cronbach óptimo para las subescalas de autoeficacia $(0,88)$ y de expectativas de resultado $(0,77)$, por lo que se aceptó la fiabilidad del modelo bifactorial (Enochs et al., 2000).

La escala de dos factores de STEBI ha sido ampliamente utilizada en publicaciones internacionales (ver por ejemplo: Bleicher, 2004; Moseley \& Utley, 2006) y, con menos tiempo en circulación, MTEBI igualmente cuenta con una importante presencia en las investigaciones científicas (ver por ejemplo: Alkhateeb, 2004; Cetinkaya \& Erbas, 2011; Moseley \& Utley, 2006; Swars, 2005). En Latinoamérica, sin embargo, se han hecho pocos estudios que intenten medir este tipo de creencias, tal vez por la aparente falta de investigación en formación inicial docente en general. Entre ellos, Cupani y Gnavi (2007) evaluaron un modelo de rendimiento académico en matemática, basado en las subescalas de expectativas de resultado en matemática y metas en matemática de la escala de autoeficacia para enseñanza media (Fouad, Smith \& Enochs, 1997), y luego llevaron a cabo dos estudios de seguimiento para mejorar las propiedades psicométricas de ambas escalas (Cupani, 2010). También se ha analizado la relación entre la autoeficacia y la solución de problemas y comunicación en universitarios de ciencias sociales (Aguirre, Muñoz, De Rueda y Blanco, 2012). Finalmente, se validó una escala de fuentes de la autoeficacia de Usher y Pajares (2009) (Zalazar, Aparicio, Ramírez y Garrido, 2011) y se evaluaron las propiedades psicométricas de un instrumento de medición de las percepciones de los docentes en relación con el desarrollo de las habilidades socioemocionales (Moreira, Pinheiro, Gomes, Cotter \& Ferreira, 2013). En Chile, el único estudio similar es uno que evaluó las propiedades psicométricas de la Escala General de Autoeficacia en la población chilena (Cid, Orellana y Barriga, 2010). En estas investigaciones no se ha utilizado STEBI-B ni MTEBI, aunque el artículo donde se valida esta última (Enochs et al., 2000) es ampliamente citado. 
Por otro lado, la fiabilidad de MTEBI ha sido muy poco explorada (Alkhateeb, 2004) y lo mismo ocurre con STEBI-B (Bleicher, 2004). Con excepción de unos pocos estudios (por ejemplo Alkhateeb, 2004; Cetinkaya \& Erbas, 2011; Moseley \& Utley, 2006), y aun cuando algunos investigadores dieron cuenta de una alta fiabilidad de las subescalas de MTEBI y STEBI-B, con alfas que van desde 0,77 hasta 0,92 para la autoeficacia, y de 0,65 a 0,76 en las expectativas de resultado (Cetinkaya $\&$ Erbas, 2011), en las investigaciones que emplean MTEBI aún se suelen citar los valores alfa reportados por Enochs y colaboradores (2000). Se requiere, por tanto, determinar la validez y fiabilidad de la escala MTEBI en distintas poblaciones y contextos (Enochs et al., 2000).

\section{Metodología de validación de la escala ECEEM}

\subsection{Procedimiento}

El presente estudio es de carácter no experimental, transversal y centrado en aspectos psicométricos, pues tiene por objetivo validar un instrumento de medida. Para su implementación se procedió a una selección no probabilística, pero heterogénea, de 12 universidades que, al año 2014, impartieran la carrera de Pedagogía Básica y que hubieran mostrado voluntad de mejorar sus procesos formativos ${ }^{5}$, además de otras dos universidades que, por el tipo de contrato de sus académicos, cercanía geográfica y experiencias previas de trabajo conjunto, garantizaban el desarrollo óptimo del trabajo propuesto. Una vez seleccionadas las universidades se aplicó la encuesta a los estudiantes de cursos de matemática de Pedagogía en Educación Básica de estas universidades.

La aplicación de la encuesta fue realizada por estudiantes becarios seleccionados en un proceso interno realizado por cada universidad participante, los que recibieron capacitación por parte del equipo de investigación.

Los criterios fueron: años de acreditación de la carrera, satisfacción de los criterios de elegibilidad para la obtención de la beca vocación de profesor, que tuvieran cuatro o más cursos del área de matemática y participaran en la prueba Inicia. 
Previamente a la aplicación del cuestionario se informó la naturaleza y responsable del estudio, se señaló que no había respuestas correctas o incorrectas y se explicó la forma de responder, enfatizando que la encuesta era anónima y que se requería del consentimiento expreso para participar. La aplicación fue autoadministrada individualmente, con el profesor a cargo del curso y el encargado de la aplicación presentes.

\subsection{Participantes}

Se trabajó con una muestra de 945 estudiantes de 49 cursos de matemática pertenecientes a 14 programas de Pedagogía en Educación Básica, impartidos en universidades de la zona norte, centro y sur de Chile. Se eligió la carrera de Pedagogía en Educación Básica pues actualmente el grueso de las asignaturas de matemática en la enseñanza básica chilena la desarrollan esos profesores.

La muestra estuvo constituida por 805 mujeres $(85,2 \%)$ y 140 hombres (14,8\%), lo que se corresponde con la distribución por sexo de esta carrera a nivel nacional (Ministerio de Educación de Chile, Mineduc, 2015), por lo que no implica un sesgo. El rango de edad fue de 18 a 30 años, con una media de 21,6 años y una desviación típica de 2,95. Del total de estudiantes, el 28,9\% egresó de la enseñanza media de un colegio municipal, el $12,6 \%$ de un particular pagado y el $58,5 \%$ de un colegio particular subvencionado (estos últimos están ligeramente sobrerrepresentados ${ }^{6}$ ). El 65,6\% cursó, previo a la aplicación del instrumento, al menos un curso de matemática y/o didáctica de la matemática, el 53\% realizó alguna mención en matemática o desea cursarla en el futuro y, aproximadamente, un $41 \%$ ha aprobado al menos una asignatura correspondiente a línea de prácticas. Además, un 27\% ha realizado clases particulares, 15,3\% prácticas en aula y $4,7 \%$ clases en aula remuneradas, lo que da cuenta de diferentes niveles de experiencia previa en docencia. Finalmente, la muestra se compuso de futuros docentes provenientes de familias con

6 Al año 2015, la matrícula de los establecimientos educativos municipales representa un $32,2 \%$ del total de la matrícula escolar, mientras que la matrícula de los establecimientos particulares pagados y subvencionados es $14,4 \%$ y $53,4 \%$, respectivamente (Mineduc, 2015). 
ingresos económicos dispares (tal como muestra la Tabla 1), lo que da cuenta de una importante heterogeneidad social en los encuestados.

Tabla 1

Porcentaje de futuros docentes por tramo de ingresos

\begin{tabular}{cc}
\hline Tramos de ingresos (en pesos chilenos) & \% futuros docentes \\
\hline 0-144.000 & 17,2 \\
144.001-288.000 & 29,3 \\
$288.001-432.000$ & 18,9 \\
$432.001-576.000$ & 10,0 \\
$576.001-720.000$ & 6,1 \\
$720.001-864.000$ & 3,2 \\
$864.001-1.008 .000$ & 3,7 \\
$1.008 .001-1.152 .000$ & 1,7 \\
$1.152 .001-1.296 .000$ & 1,0 \\
$1.296 .001-1.440 .000$ & 1,1 \\
$1.440 .001-1.584 .000$ & 1,2 \\
1.584 .001 o más & 6,6 \\
\hline
\end{tabular}

Fuente: Elaboración propia.

\subsection{Instrumento}

Para medir las creencias de eficacia en la enseñanza de la matemática se dispuso de la Escala de Creencias de Eficacia en la Enseñanza de la Matemática (ECEEM). Este instrumento consta de 21 ítems tipo Likert de cinco opciones de respuesta, que implican diferentes grados de acuerdo con cada afirmación y que van desde 1 (muy en desacuerdo) a 5 (muy de acuerdo). Además, la escala cuenta con dos dimensiones correlacionadas entre sí:

- 13 ítems miden la Autoeficacia en la Enseñanza de la Matemática (AEM);

- 8 ítems miden las Expectativas de Resultado en la Enseñanza de la Matemática (EREM) ${ }^{7}$.

La consigna inicial a los estudiantes fue "Respecto de sus creencias y percepciones, para cada una de las afirmaciones indique su

7 Ver en el Anexo los ítems ECEEM. 
grado de acuerdo. Considere la siguiente escala: Muy en desacuerdo (1); En desacuerdo (2); Ni de acuerdo ni en desacuerdo (3); De acuerdo (4); Muy de acuerdo (5)".

El cuestionario incluyó, además, algunas variables sociodemográficas y otras de caracterización académica. Entre las preguntas de identificación sociodemográfica se incluyen el sexo y la edad. Dentro de las preguntas de caracterización académica se encuentran el tipo de administración del establecimiento del que egresó de cuarto año medio (municipal, subvencionado o particular; técnico profesional o científico humanista), la universidad en la que estudia, el año de ingreso a la carrera y el año que está cursando. Se preguntó también si está cursando o pretende cursar una mención en matemática, y las preferencias del estudiante entre distintos sectores del aprendizaje (Lenguaje, Matemática, Ciencias Sociales y Ciencias Naturales).

\subsection{Análisis estadístico}

En esta investigación se realizó la validación convergente y factorial de la Escala de Creencias de Eficacia en la Enseñanza de la Matemática (ECEEM) y una descripción de los sujetos según los puntajes obtenidos en ella. Si bien para la validación de la escala se mantuvieron las puntuaciones originales, al describir a los sujetos se invirtieron los puntajes de los ítems que tenían una redacción inversa $(-)$, recodificando los valores asignados a estos ítems ( 1 se transformó en $5 ; 2$ en $4 ; 4$ en 2 ; y 5 en 1 ), de manera que para todos ellos una mayor puntuación indicara creencias más positivas respecto de la capacidad de enseñar matemáticas.

El análisis contempló cinco fases (las primeras cuatro corresponden a la validación del instrumento):

- análisis de la discriminación de los ítems;

- análisis de la estructura factorial de la escala;

- determinación de la fiabilidad de cada una de las subescalas;

- análisis de la validez concurrente de la escala; y

- descripción de los sujetos según los puntajes obtenidos en la ECEEM definitiva. 
En la fase de análisis de la discriminación de los ítems se reportaron las propiedades psicométricas exhibidas por cada ítem según sus medias, sus no respuestas, sus desviaciones típicas, sus asimetrías y sus curtosis.

Con el fin de estimar el ajuste del modelo hipotetizado en los datos, se realizó un análisis factorial confirmatorio (AFC). Aprovechando que se conocía el modelo original de la escala Mathematics Teaching Efficacy Belief Instrument (MTEBI) (Enochs et al., 2000), se puso a prueba un modelo bifactorial, que contempló la correlación entre los factores de la escala ECEEM: Autoeficacia en la Enseñanza de la Matemática (AEM) y Expectativas de Resultado en la Enseñanza de la Matemática (EREM). La expresión visual del modelo se puede observar en la Figura 1.

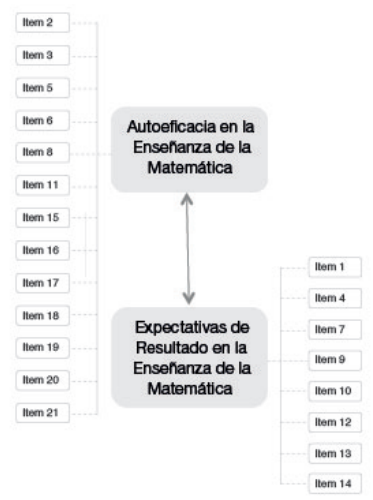

Figura 1. Escala Mathematics Teaching Efficacy Belief Instrument.

Fuente: Modelo MTEBI (Enochs et al., 2000).

El AFC se realizó mediante el procedimiento de estimación de mínimos cuadrados no ponderados (ULS) sobre las correlaciones tetracóricas entre los ítems, pues se ha demostrado que ese procedimiento de estimación (Forero, Maydeu-Olivares \& GallardoPujol, 2009) y ese tipo de correlaciones (Flora \& Curran, 2004; Holgado-Tello, Chacón-Moscoso, Barbero-García \& Vila-Abad, 2010) permiten obtener resultados óptimos cuando se trabaja con variables ordinales, al reconocer la naturaleza ordinal de las variables observadas (Asún, Navarro \& Alvarado, 2015). Esta versión del AFC es llamada también Análisis Factorial de Ítems (AFI) por algunos 
160 VALIDACIÓN DE LA ESCALA DE CREENCIAS DE EFICACIA EN LA ENSEÑANZA DE LA MATEMÁTICA (ECEEM) Y CARACTERIZACIÓN DE LAS CREENCIAS DE ESTUDIANTES DE PEDAGOGÍA BÁSICA - M. Verdugo, R. Asún y S. Martínez

autores (Wirth \& Edwards, 2007). Para estos análisis se empleó el software M-Plus 7.

Habiendo estimado el nivel de correlación entre las subescalas AEM y EREM, se evaluó la fiabilidad de cada subescala por separado, empleando el estadístico alfa ordinal (Elosua y Zumbo, 2008). Se consideró aceptable un alfa ordinal mayor a 0,8.

Para determinar la validez externa de la escala, se analizó la relación entre las subescalas AEM y EREM con las respuestas dadas por los sujetos a distintas preguntas relacionadas teóricamente con la autoeficacia y las expectativas de resultado. La asociación de las subescalas con las variables cuantitativas se analizó a partir de estadístico $r$ de Pearson $(r)$, mientras que para la relación con las variables dicotómicas se utilizó la correlación biserial puntual $\left(\mathrm{r}_{\mathrm{bp}}\right)$.

Habiendo determinado la estructura y los ítems que finalmente compondrían la escala ECEEM, se dio cuenta de la distribución de los puntajes obtenidos por los sujetos que respondieron el cuestionario. Para interpretar los puntajes, se sumaron las puntuaciones obtenidas en cada ítem retenido, y luego se dividió esa puntuación por el número de ítems retenidos.

\section{Resultados}

\subsection{Validación del instrumento}

\subsubsection{Análisis de la discriminación de los ítems}

Tal como se observa en la Tabla 2, las propiedades psicométricas de los ítems de la Escala de Creencias de Eficacia en la Enseñanza de la Matemática (ECEEM) en general son buenas. Ningún ítem concentra más del 1,3\% de no respuestas. En relación con los promedios obtenidos, la mayoría de los ítems tiene un valor intermedio y, junto con ello, las desviaciones típicas son lo suficientemente altas como para afirmar que la mayoría de las preguntas discrimina entre los distintos sujetos. Por otro lado, si analizamos la curtosis y la asimetría, la gran mayoría de los ítems posee una distribución mesocúrtica y simétrica (los coeficientes están entre - 0,5 y 0,5), dando cuenta de 
asimetrías o curtosis moderadas, mostrando que las respuestas a los ítems no se distribuyen concentradamente.

Tabla 2

Propiedades psicométricas items de la ECEEM

\begin{tabular}{cccccc}
\hline$N^{0}$ Ítem & \% de No Respuestas & Media & Desv. Tip. & Asimetría & Curtosis \\
\hline I1 & 0,6 & 3,3 & 1,06 & $-0,25$ & $-0,37$ \\
I2 & 0,4 & 4,4 & 0,86 & $-1,35$ & $\mathbf{1}, 64$ \\
I3 & 0,6 & 2 & 1,27 & $-1,03$ & $-0,07$ \\
I4 & 0,6 & 4,2 & 0,86 & $-0,95$ & 0,64 \\
I5 & 0,5 & 3 & 1,01 & $-0,16$ & $-0,4$ \\
I6 & 1,3 & 2 & 1,03 & $-0,72$ & $-0,19$ \\
I7 & 0,7 & 3,7 & 1,15 & $-0,63$ & $-0,36$ \\
I8 & 0,6 & 1,6 & 0,93 & $-1,76$ & 2,76 \\
I9 & 0,7 & 4,4 & 0,82 & $-1,76$ & 3,72 \\
I10 & 0,6 & 3,7 & 0,92 & $-0,43$ & $-0,08$ \\
I11 & 1,1 & 3,3 & 1,01 & $-0,35$ & $-0,29$ \\
I12 & 0,8 & 3,5 & 0,95 & $-0,27$ & $-0,26$ \\
I13 & 0,7 & 3,8 & 0,9 & $-0,47$ & $-0,01$ \\
I14 & 1,1 & 3,7 & 0,97 & $-0,44$ & $-0,16$ \\
I15 & 0,7 & 2 & 1,07 & $-0,91$ & 0,14 \\
I16 & 1 & 3,7 & 0,95 & $-0,51$ & $-0,07$ \\
I17 & 1,3 & 3,5 & 1,22 & 0,44 & $-0,73$ \\
I18 & 1,1 & 2,1 & 1,24 & $-0,88$ & $-0,23$ \\
I19 & 0,7 & 1,9 & 1,06 & -1 & 0,16 \\
I20 & 1,1 & 4,7 & 0,73 & $-2,95$ & 9,9 \\
I21 & 1 & 2,2 & 1,11 & $-0,75$ & $-0,13$ \\
\hline
\end{tabular}

Fuente: Elaboración propia.

Como se observa en la Tabla 2, los ítems 2, 8, 9 y 20 constituyen excepciones a esas favorables propiedades: todos ellos presentaron respuestas muy homogéneas. Por un lado, los ítems 2 (media 4,4), $9($ media $=4,4 ;$ curtosis $=3,72)$ y $20($ media $=4,7 ;$ curtosis $=9,9)$ mostraron ser ítems muy deseables para los sujetos, concentrando sus respuestas en los valores más altos. Por otro lado, el ítem 8 (media $=$ 1,6 ; curtosis $=2,76$ ) mostró ser un ítem muy poco deseable.

Sin embargo, ninguno de los ítems anteriores alcanza niveles completamente negativos, por lo que se decidió, provisionalmente, no eliminar ninguno de ellos hasta corroborar su funcionamiento en el análisis factorial. 
162 VALIDACIÓN DE LA ESCALA DE CREENCIAS DE EFICACIA EN LA ENSEÑANZA DE LA MATEMÁTICA (ECEEM) Y CARACTERIZACIÓN DE LAS CREENCIAS DE ESTUDIANTES DE PEDAGOGÍA BÁSICA - M. Verdugo, R. Asún y S. Martínez

\subsubsection{Estructura factorial de la escala ECEEM}

Para determinar si los datos empíricos se ajustan al modelo teórico sobre la base del cual se ha diseñado el instrumento, se evaluó el modelo bifactorial original en función de las siguientes medidas de bondad de ajuste: $\chi^{2}$, RMSEA, CFI y TLI. El análisis factorial arrojó un ajuste no óptimo de la estructura hipotetizada $\left(\chi^{2}{ }_{188}=1348,0 p<\right.$ 0,$00 ; \chi^{2} / g .1 .=7,2 ;$ RMSEA $\left.=0,081 ; \mathrm{CFI}=0,86 ; \mathrm{TLI}=0,85\right)^{8}$, es decir, los parámetros estimados no lograron reproducir los datos reales. Por otro lado, en relación con la calidad de los ítems, todos mostraron cargas factoriales aceptables con su factor $(\lambda>0,4)$, a excepción del ítem $17(\lambda=0,26)$ que debió ser eliminado del modelo. Además, se evidenció una correlación positiva y significativa $(r=0,2)$ entre las subescalas Autoeficacia en la Enseñanza de la Matemática (AEM) y Expectativas de Resultado en la Enseñanza de la Matemática (EREM) aunque no excesivamente alta, por lo que se aceptó la hipótesis respecto de la estructura de dos factores de la escala.

Posteriormente, se verificó cuánto mejora el ajuste del modelo eliminando el ítem 17, encontrándose que los valores de los distintos estadísticos de ajuste se mantuvieron prácticamente iguales $\left(\chi_{169}^{2}=\right.$ $1250,1 p<0,00 ; \chi^{2} / g .1 .=7,4 ;$ RMSEA $=0,082 ; \mathrm{CFI}=0,87 ; \mathrm{TLI}=$ $0,85)$, al igual que las cargas factoriales de los distintos ítems $(\lambda>0,4)$ y la correlación entre las dimensiones de la escala $(r=0,2)$.

El análisis de los índices de modificación de este segundo modelo mostró que los ítem 5 y 11 compartían demasiada varianza que no era explicada por su factor común, por lo que se decidió eliminar el ítem 11 por tener una carga factorial un poco menor $(\lambda=0,553)$.

El ajuste del modelo bifactorial con los ítems 11 y 17 eliminados mejoró bastante pues, aun cuando $\chi^{2}$ arrojó un valor insatisfactorio $\left(\chi^{2}{ }_{151}=804,6 p<0,00 ; \chi^{2} / g .1 .=5,3\right)$, RMSEA, CFI y TLI mostraron rangos bastante mejores $(0,068 ; 0,91$ y 0,9 , respectivamente). Todos los ítems obtuvieron cargas factoriales

8 Para hablar de un ajuste óptimo se debe obtener $\chi^{2} / \mathrm{gl}<3$ (Kline, 2005); RMSEA $<0,07$ (Steiger, 2007); CFI y TLI > 0,095 (Hu \& Bentler, 1999). 
satisfactorias con su factor $(\lambda \geq 0,4)$ y la correlación entre las dimensiones disminuyó $(r=0,19)$. Se evidenció así la validez por estructura interna del modelo bifactorial hipotetizado con solo la eliminación de dos ítems.

Para mejorar la bondad de ajuste del modelo y hacerlo más parsimonioso, se analizaron nuevamente los índices de modificación. En ellos se observó una fuerte carga potencial del ítem 2 con las dos dimensiones del instrumento, lo que producía un importante desajuste de $\chi^{2}$, por lo que se resolvió eliminarlo.

En consecuencia, se replicó el AFC eliminando los ítems 2, 11 y 17 , encontrándose un ajuste óptimo del modelo según algunos los indicadores (RMSEA), y valores muy cercanos a ese límite en otros (CFI y TLI), aunque aún un poco altos en $\chi^{2}\left(\chi_{134}^{2}=659,9 p<0,00\right.$; $\chi^{2}$ /g.l. $=4,9 ;$ RMSEA $\left.=0,064 ; \mathrm{CFI}=0,93 ; \mathrm{TLI}=0,92\right)$, lo que no se considera tan problemático en muestras grandes como la empleada en esta investigación, pues $\chi^{2}$ es muy sensible en esas situaciones. Junto con ello, las cargas factoriales se mantuvieron aceptables $(\lambda>0,4)$ y, de acuerdo con lo esperado, la correlación entre las dos dimensiones de la escala fue menor $(r=0,16)$, pues el ítem 2 medía ambas dimensiones a la vez. En consecuencia, pese a no tener un ajuste completamente óptimo, se ha decidido mantener esta como la estructura final del instrumento.

Las malas propiedades psicométricas de los ítems eliminados coinciden con problemas en sus fraseos. En el caso del ítem 2, que ya había presentado deficiencias para discriminar entre los distintos sujetos encuestados, exhibe una redacción muy abstracta e hipotética (continuamente voy a encontrar mejores formas para enseñar matemática), que explica su relación con ambas dimensiones del instrumento. Por otro lado, el ítem 11 muestra un fraseo (comprendo conceptos matemáticos lo suficientemente bien como para ser efectivo al enseñar matemática elemental) muy parecido al del ítem 5 (sé cómo enseñar conceptos matemáticos de forma efectiva), llevando a que ambos compartan una varianza demasiado alta. Finalmente, el ítem 17 está redactado de manera tal que no permite que el encuestado plantee sus creencias acerca de las habilidades que posee para enseñar 
matemática (me pregunto si tendré las habilidades necesarias para enseñar matemática), sino que más bien lleva a que el sujeto manifieste si se ha cuestionado en torno a estas habilidades. Esto último no responde a la definición de ninguna de las dimensiones de la escala, por lo que parece lógico que este ítem tenga una baja carga factorial con su factor.

De este modo, la escala definitiva se constituyó por 18 ítems (ocho ítems en EREM y 10 ítems en AEM), tal como muestra la Figura 2:

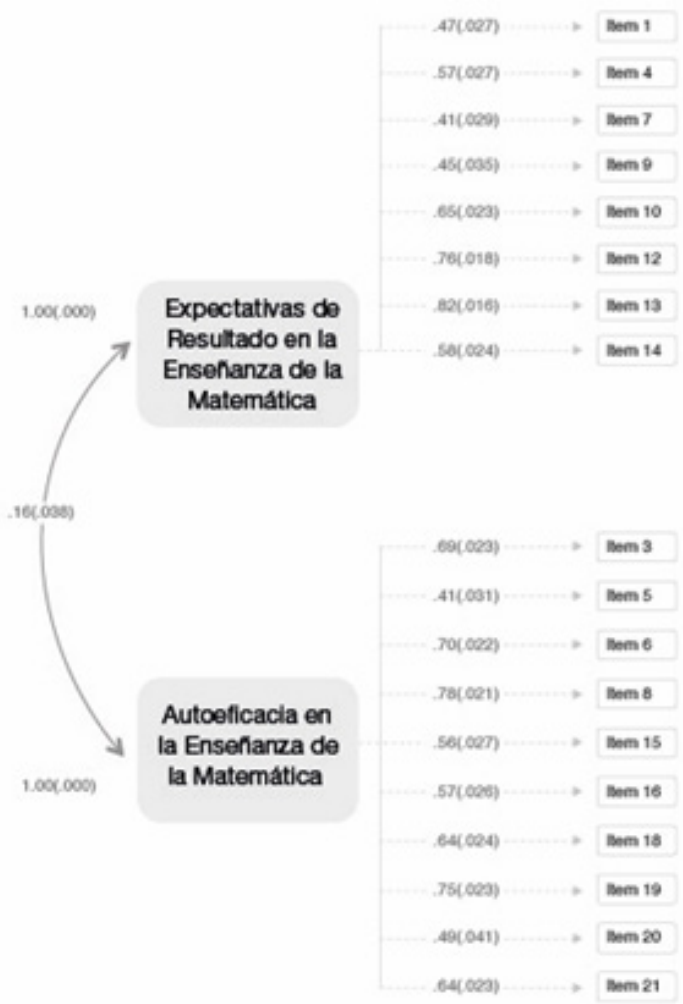

Figura 2. Escala de Creencias de Eficacia en la Enseñanza de la Matemática Fuente: Elaboración propia.

\subsubsection{Fiabilidad de las subescalas AEM y EREM}

Debido a la baja correlación $(r=0,16)$ entre las subescalas Autoeficacia en la Enseñanza de la Matemática (AEM) y Expectativas de Resultado 
en la Enseñanza de la Matemática (EREM) fue conveniente estimar la fiabilidad de las subescalas por separado.

La fiabilidad de la subescala EREM presentó un alfa ordinal de 0,81, mientras que AEM mostró una fiabilidad de 0,86, lo que da cuenta de una muy adecuada consistencia interna de ambas subescalas.

\subsubsection{Validez concurrente de la escala ECEEM}

Para aportar evidencias de la validez del instrumento se correlacionaron las puntuaciones obtenidas por los sujetos en ambas subescalas (AEM y EREM) con diferentes variables que teóricamente debieran o no estar asociadas con cada una de ellas. Del total de variables externas introducidas en el análisis, AEM debía estar significativamente correlacionada con aquellas que se refirieran a la obtención de una mayor experiencia docente, aprobación en cursos de matemática y prácticas en aula, y con las autopercepciones acerca del nivel de preparación que poseen en distintas subdisciplinas de la matemática. Este tipo de experiencias, al conllevar una mayor familiarización con la matemática y su enseñanza, es probable que fortalezcan la autopercepción de los estudiantes en relación a sus capacidades para enseñar. En cambio, en el caso de EREM, se esperaba que no se correlacionase con ninguna de las variables introducidas, porque esa subescala mide una expectativa más sistémica, que probablemente ese tipo de experiencias individuales no sean capaces de modificar.

Como se muestra en las Tablas 3 y 4 , se confirmaron las expectativas previas: mientras la subescala AEM presentó una relación directa y significativa con las variables esperadas, la subescala EREM es insensible a la gran mayoría de ellas ${ }^{9}$. De este modo, ambas subescalas presentaron evidencia de validez externa.

Con las dos variables que sí poseen una correlación significativa, esta es de muy baja intensidad. 
166 VALIDACIÓN DE LA ESCALA DE CREENCIAS DE EFICACIA EN LA ENSEÑANZA DE LA MATEMÁTICA (ECEEM) Y CARACTERIZACIÓN DE LAS CREENCIAS DE ESTUDIANTES DE PEDAGOGÍA BÁSICA - M. Verdugo, R. Asún y S. Martínez

Tabla 3

Correlaciones biseriales puntuales entre las subescalas AEM y EREM y variables categóricas externas

\begin{tabular}{|c|c|c|c|c|}
\hline \multirow{3}{*}{ Variable } & \multirow{2}{*}{\multicolumn{2}{|c|}{$\begin{array}{c}\text { Subescala } \\
\text { AEM }\end{array}$}} & \multirow{2}{*}{\multicolumn{2}{|c|}{$\begin{array}{l}\text { Subescala } \\
\text { EREM }\end{array}$}} \\
\hline & & & & \\
\hline & $r_{\mathrm{bp}}$ & $p$ & $r_{\mathrm{bp}}$ & $P$ \\
\hline Sexo & 0,009 & 0,785 & 0,005 & 0,875 \\
\hline $\begin{array}{l}\text { Administración del establecimiento } \\
\text { origen: particular pagado. }\end{array}$ & $-0,005$ & 0,882 & 0,013 & 0,704 \\
\hline $\begin{array}{l}\text { Administración del establecimiento } \\
\text { origen: particular subvencionado. }\end{array}$ & 0,02 & 0,54 & 0,019 & 0,576 \\
\hline $\begin{array}{l}\text { Administración del establecimiento } \\
\text { origen: municipal. }\end{array}$ & $-0,023$ & 0,483 & $-0,045$ & 0,181 \\
\hline $\begin{array}{l}\text { Programa de formación de enseñanza } \\
\text { media. }\end{array}$ & $-0,002$ & 0,94 & 0,064 & 0,056 \\
\hline $\begin{array}{l}\text { Tener experiencia haciendo clases (en } \\
\text { general). }\end{array}$ & 0,315 & $0,001^{* * * *}$ & 0,069 & $0,038^{*}$ \\
\hline $\begin{array}{l}\text { Tener experiencia haciendo clases } \\
\text { particulares. }\end{array}$ & 0,29 & $0,001^{* * * *}$ & 0,033 & 0,318 \\
\hline $\begin{array}{l}\text { Tener experiencia haciendo clases en aula } \\
\text { (práctica profesional). }\end{array}$ & 0,15 & $0,001^{* * *}$ & 0,041 & 0,223 \\
\hline $\begin{array}{l}\text { Tener experiencia haciendo clases en aula } \\
\text { (trabajo remunerado). }\end{array}$ & 0,169 & $0,001^{* * *}$ & 0,049 & 0,138 \\
\hline $\begin{array}{l}\text { Cursar una mención en matemática } \\
\text { actualmente. }\end{array}$ & 0,028 & 0,409 & $-0,062$ & 0,063 \\
\hline $\begin{array}{l}\text { Deseo de cursar una mención en } \\
\text { matemática en el futuro. }\end{array}$ & 0,057 & 0,142 & 0,016 & 0,682 \\
\hline
\end{tabular}

Notas: En negrilla, correlación significativa.

* La correlación es significativa al nivel 0,05 (bilateral).

** La correlación es significativa al nivel 0,01 (bilateral).

*** La correlación es significativa al nivel 0,001 (bilateral).

Fuente: Elaboración propia

Tabla 4

Correlaciones de Pearson entre las subescalas AEM y EREM y variables cuantitativas externas

\begin{tabular}{lcccc}
\hline & \multicolumn{2}{c}{ Subescala AEM } & \multicolumn{2}{c}{ Subescala EREM } \\
\hline Variable & $r$ & $P$ & $r$ & $P$ \\
\hline $\begin{array}{l}\text { Cantidad de cursos aprobados de } \\
\text { matemática y/o didáctica de la matemática. }\end{array}$ & $\mathbf{0 , 2 9}$ & $\mathbf{0 , 0 0 \mathbf { 1 } ^ { * * * }}$ & 0,032 & 0,352 \\
\hline $\begin{array}{l}\text { Cantidad de prácticas en aula aprobadas. } \\
\text { Nivel de preparación percibido en }\end{array}$ & $\mathbf{0 , 1 4 9}$ & $\mathbf{0 , 0 0 \mathbf { 1 } ^ { * * * }}$ & 0,051 & 0,154 \\
$\begin{array}{l}\text { Álgebra. } \\
\begin{array}{l}\text { Nivel de preparación percibido en Datos } \\
\text { yzar. }\end{array}\end{array}$ & $\mathbf{0 , 2 4 1}$ & $\mathbf{0 , 0 0 1}^{* * *}$ & 0,011 & 0,736 \\
$\begin{array}{l}\text { Nivel de preparación percibido en } \\
\text { Geometría. }\end{array}$ & $\mathbf{0 , 2 9 9}$ & $\mathbf{0 , 0 0 1}^{* * * *}$ & 0,019 & 0,576 \\
\hline $\begin{array}{l}\text { Nivel de preparación percibido en } \\
\text { Números. }\end{array}$ & $\mathbf{0 , 3 3 5}$ & $\mathbf{0 , 0 0 \mathbf { 1 } ^ { * * * }}$ & $\mathbf{0 , 0 6 9}$ & $\mathbf{0 , 0 4 0}$ \\
\hline
\end{tabular}

Notas: En negrilla la correlación significativa.

* La correlación es significativa al nivel 0,05 (bilateral).

** La correlación es significativa al nivel 0,01 (bilateral).

*** La correlación es significativa al nivel 0,001 (bilateral).

Fuente: Elaboración propia. 
4.2. Distribución de las puntuaciones de los sujetos en la Escala ECEEM

El cálculo de los puntajes de los estudiantes de Pedagogía Básica en la Escala de Creencias de Eficacia en la Enseñanza de la Matemática (ECEEM) se hizo tomando en cuenta los 18 ítems retenidos, considerando un rango de 1 ("creencias muy negativas") a 5 ("creencias muy positivas").

Como se observa en la Tabla 5, la media de los sujetos en la subescala Autoeficacia en la Enseñanza de la Matemática (AEM) es de 3,95; mientras que en la subescala Expectativas de Resultado en la Enseñanza de la Matemática (EREM) es de 3,78, y la desviación típica en ambos casos es de 0,6 puntos. Vale decir, los participantes tienen creencias ligeramente positivas en ambas subescalas, sobre todo en AEM, donde una cantidad importante de sujetos se encuentra entre los valores 4 y 5 de la subescala, tal como se observa en la Figura 3. En el mismo sentido, la distribución de EREM y AEM en deciles (ver Tabla 5), da cuenta que al menos un 60\% y 40\% de los individuos presenta creencias que están por debajo del valor 4 (ligeramente positivas), respectivamente en ambas subescalas. En consecuencia, los futuros docentes son más escépticos respecto de las posibilidades generales de producir logros significativos en sus futuros alumnos y más optimistas respecto de sus propias capacidades como docentes.

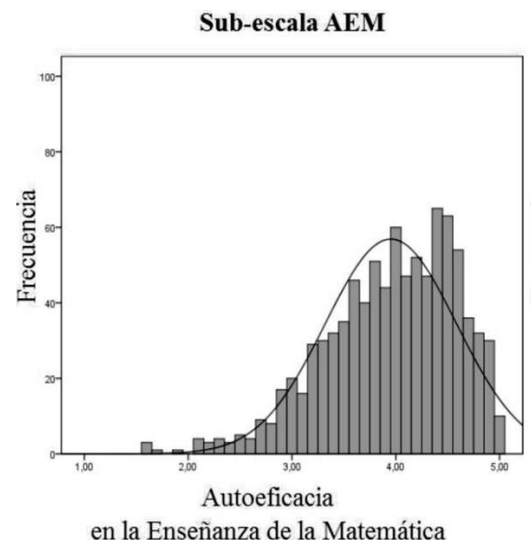

Figura 1

Fuente: Elaboración propia.

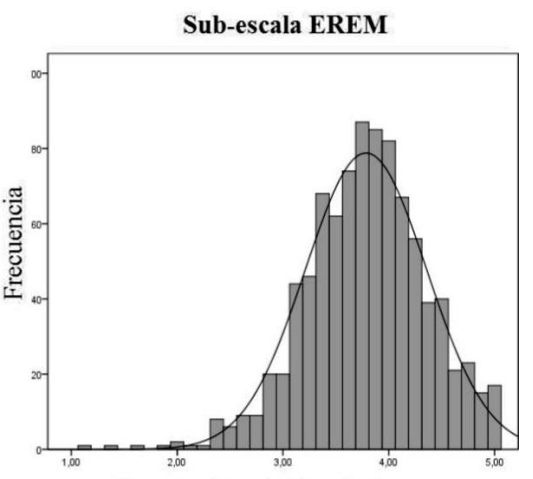

Expectativas de Resultado en la Enseñanza de la Matemática 
Por otro lado, en los histogramas se observa una distribución asimétrica negativa de los datos en AEM y una distribución simétrica en EREM, lo que es corroborado por el estadístico de asimetría (ver Tabla 6) que presenta un valor menor a $-0,5$ en AEM y entre $-0,5$ y 0,5 en EREM. Además, las puntuaciones en ambas subescalas poseen una distribución mesocúrtica y leptocúrtica, respectivamente.

Tabla 5

Media, desviación típica y distribución por deciles de los valores en escalas EREM y AEM

\begin{tabular}{lccc}
\hline & & Subescala EREM & Subescala AEM \\
\hline $\mathrm{N}$ & & 906 & 901 \\
\hline Media & 3,78 & 3,95 \\
\hline Desv. típ. & 0,6 & 0,6 \\
\hline \multirow{4}{*}{ Percentiles } & 10 & 3,13 & 3,1 \\
& 20 & 3,38 & 3,4 \\
& 30 & 3,5 & 3,66 \\
& 40 & 3,63 & 3,8 \\
& 50 & 3,75 & 4 \\
& 70 & 3,88 & 4,2 \\
& 80 & 4,13 & 4,4 \\
& 90 & 4,25 & 4,5 \\
& & 4,5 & 4,7 \\
\hline
\end{tabular}

Fuente: Elaboración propia.

Tabla 6

Asimetría y curtosis en escalas EREM y AEM

\begin{tabular}{lcc}
\hline & Subescala EREM & Subescala AEM \\
\hline Asimetría & $-0,38$ & $-0,702$ \\
Error típ. de asimetría & 0,081 & 0,081 \\
Curtosis. & 0,802 & 0,345 \\
Error típ. de curtosis. & 0,162 & 0,163 \\
\hline
\end{tabular}

Fuente: Elaboración propia.

En síntesis, si bien son pocos los estudiantes de Pedagogía en Educación Básica en Chile que poseen AEM y EREM negativas, los juicios que tienen respecto de los aprendizajes que puede generar su futuro desempeño profesional tienden a ser solo ligeramente positivos. En este sentido, los estudiantes de Pedagogía Básica se encuentran en una situación que está entre la falta de expectativas y una moderada tendencia hacia expectativas positivas respecto de los aprendizajes en matemática que podrán generar en el futuro. 
Específicamente en relación con AEM, se evidencia que, aun cuando una cantidad considerable de los encuestados percibe que sus capacidades y habilidades les permitirán actuar eficazmente sobre el aprendizaje de sus futuros estudiantes, un grupo minoritario pero importante obtiene puntuaciones bajas, lo que podría generar profecías autocumplidas negativas en su desempeño laboral futuro.

En relación con EREM, los puntajes obtenidos por los estudiantes de Pedagogía Básica son en promedio menos positivos que para la escala AEM, por lo que hay menos de ellos que se encuentran en los valores más altos de la subescala. En consecuencia, predominan creencias neutras o que indican solo débiles expectativas acerca de la eficacia de la enseñanza para provocar un aprendizaje significativo en los estudiantes, lo que posiblemente también influirá negativamente en sus desempeños futuros.

\section{Conclusiones}

A partir de la evaluación de los atributos psicométricos de la Escala de Creencias de Eficacia en la Enseñanza de la Matemática (ECEEM), es posible afirmar que hay evidencias de su validez convergente y factorial, de la buena capacidad de discriminación de sus ítems y de la alta fiabilidad de la versión final de la escala. Se confirma así el ajuste de la estructura bifactorial hipotetizada a partir de la escala original Mathematics Teaching Efficacy Belief Instrument (MTEBI) (Enochs et al., 2000), aunque para ello debieron ser eliminados tres ítems.

En consecuencia, es posible afirmar que la versión final de la escala ECEEM compuesta por 18 ítems constituye un instrumento fiable y válido para evaluar las creencias de eficacia en la enseñanza de la matemática de los futuros docentes chilenos a partir de dos subescalas: la Autoeficacia en la Enseñanza de la Matemática (AEM) y las Expectativas de Resultado en la Enseñanza de la Matemática (EREM), compuestas por 10 y ocho ítems respectivamente. En conjunto, este instrumento puede ser empleado para medir las creencias que tienen los estudiantes de Pedagogía en Educación Básica respecto de los aprendizajes matemáticos que puede generar su futuro desempeño profesional. 
170 VALIDACIÓN DE LA ESCALA DE CREENCIAS DE EFICACIA EN LA ENSEÑANZA DE LA MATEMÁTICA (ECEEM) Y CARACTERIZACIÓN DE LAS CREENCIAS DE ESTUDIANTES DE PEDAGOGÍA BÁSICA - M. Verdugo, R. Asún y S. Martínez

En relación con la distribución de las puntuaciones en los estudiantes de Pedagogía en Educación Básica encuestados se observó que, aun cuando son pocos aquellos que obtienen puntajes negativos, en promedio sus creencias son solo ligeramente positivas, pues la mayoría no alcanza los valores más altos en ninguna de las subescalas. Además se evidenció que los futuros docentes son más optimistas con respecto a sus propias capacidades (autoeficacia) que en la capacidad social de la educación (expectativas de resultado), lo que se puede relacionar con una suerte de omnipotencia del pensamiento juvenil (Aberastury, 1971), donde los jóvenes se insertan en los desafíos de la vida social adulta con mayor confianza en sus propias capacidades que en las herramientas que brinda el contexto.

Que la subescala AEM no alcance niveles totalmente satisfactorios puede relacionarse con que los sujetos encuestados no son estudiantes de Pedagogía en Matemática y no necesariamente tienen intención de serlo, por lo que podrían percibir que poseen más habilidades para enseñar otras áreas del conocimiento. Aun así, considerando que más de un $70 \%$ de los encuestados señalaron estar motivados con el estudio de la matemática, que los profesores de enseñanza básica en su desempeño laboral están expuestos a enseñar cualquier asignatura y que la matemática es una de las asignatura principales dentro del currículo escolar chileno (Mineduc, 2016), es necesario resaltar que la formación actual de los futuros docentes no propicia en ellos una mayor confianza en sus habilidades para enseñar esta área del conocimiento.

Junto con lo anterior, al menos un $60 \%$ de sujetos que está preparándose para ejercer la docencia, duda de las potencialidades de la educación matemática para poder generar cambios significativos en el aprendizaje de los estudiantes (EREM). Si bien la poca confianza en la capacidad de la educación matemática puede comprenderse en un contexto de desprestigio general de la Pedagogía, originado en la dictadura (Ávalos, 2004), es de extrañar que habiendo pasado más de 30 años Chile no cuente con un sistema educativo que sea capaz de mejorar las expectativas en torno a la docencia, al menos en los futuros profesores. 
Finalmente, si consideramos que son las creencias positivas las que traen aparejadas consecuencias educacionales positivas (utilización de una mayor variedad de estrategias de enseñanza (Wenta, 2000), manejo de investigaciones y estrategias de enseñanza centradas en el estudiante (Czerniak, 1990), uso de técnicas de enseñanza más difíciles de implementar (Czerniak \& Lumpe, 1996)), se puede observar que hoy no son muchos los estudiantes de Pedagogía en Educación Básica que presentan creencias que aseguren un desempeño óptimo en su futura labor como docentes.

Estos hallazgos dan pie para profundizar en el estudio de variables subjetivas críticas para el actual y futuro desempeño docente, incorporando las creencias en la eficacia de la enseñanza, y junto con ello, abrir la discusión acerca del tipo de sistemas de evaluación de calidad docente necesarios de desarrollar. En este sentido, parece relevante profundizar en el estudio de la relación de las CREEM con variables como la ansiedad matemática, la vocación por el estudio y enseñanza de la matemática, con escalas que midan el aprendizaje y desempeño docente y las creencias acerca del aprendizaje de la matemática. Todas relaciones esperables según la evidencia empírica y teórica presentada anteriormente.

También se hace necesario que se validen nuevas escalas que midan diferentes constructos subjetivos relacionados con la docencia, para poder diagnosticar y monitorear los procesos formativos de las universidades que forman profesores, así como aquellos correspondientes a instancias de desarrollo profesional docente.

\section{Referencias}

Aberastury, A. (1971). Adolescencia. Buenos Aires: Ediciones Kargieman.

Aguirre, J. F., Muñoz, F., de Rueda, B., y Blanco, J. R. (2012). Composición factorial de una escala de autoeficacia en la solución de problemas y comunicación en universitarios de ciencias sociales. Formación universitaria, 5(5), 27-38.

https://doi.org/10.4067/s0718-50062012000500004 
172 VALIDACIÓN DE LA ESCALA DE CREENCIAS DE EFICACIA EN LA ENSEÑANZA DE LA MATEMÁTICA (ECEEM) Y CARACTERIZACIÓN DE LAS CREENCIAS DE ESTUDIANTES DE PEDAGOGÍA BÁSICA - M. Verdugo, R. Asún y S. Martínez

Alkhateeb, H. M. (2004). Internal consistency reliability and validity of the Arabic translation of the mathematics teaching efficacy beliefs instrument. Psychological Reports, 3(94), 833-838. https://doi.org/10.2466/pr0.94.3.833-838

Ashton, P. (1985). Motivation and the teachers' sense of efficacy. En C. Ames $\&$ R. Ames (Eds.), Research motivation in education: The classroom milieu, Vol. 2. (pp. 141-174). New York: Academic Press.

Ashton, P., Buhr, D., \& Crocker, L. (1984). Teachers' sense of efficacy: A selfor norm-referenced construct? Florida Journal of Educational Research, 26(1), 29-41.

Asún, R. A., Navarro, K., \& Alvarado, J. M. (2015). Developing multidimensional Likert scales using item factor analysis the case of four-point items. Sociological Methods \& Research, 1(45), 109-133. https://doi.org/10.1177/0049124114566716

Ávalos, B. (2004). La formación docente inicial en Chile. Santiago de Chile. Recuperado de http://www.ub.edu/obipd/PDF\%20docs/Aspectes\%20laborals/ Documents/La\%20Formacion\%20Docente\%20Inicial\%20en\%20 Chile.\%20AVALOS.pdf

Bandura, A. (1977). Self-efficacy: Toward a unifying theory of behavioral change. Psychological Review, 2(84), 191-215. https://doi.org/10.1037//0033-295x.84.2.191

Bandura, A. (1978). Reflections on self-efficacy. Advances in Behaviour Research and Therapy, 4(1), 237-269. https://doi.org/10.1016/0146-6402(78)90012-7

Bandura, A. (1984). Recycling misconceptions on perceived selfefficacy. Cognitive Therapy and Research, 3(8), 231-255. https://doi.org/10.1007/bf01172995

Bandura, A. (1986). Social foundations of thought and action: A social cognitive theory. New Jersey: Prentice Hall.

Bandura, A. (1995). On rectifying conceptual ecumenism. En J. E. Maddux (Ed.), Self-efficacy, adaptation, and adjustment: Theory, research, and application (pp. 347-375). New York: Plenum.

Bandura, A. (1997). Self-efficacy: The exercise of control. New York: Freeman.

Bandura, A. (1998). Health promotion from the perspective of social cognitive theory. Psychology and Health, 4(13), 623-649. https://doi.org/10.1080/08870449808407422 
Bandura, A. (2004). Health promotion by social cognitive means. Health Education and Behavior, 2(31), 143-164.

https://doi.org/10.1177/1090198104263660

Bandura, A. (2006). Toward a psychology of human agency. Perspectives on Psychological Science, 2(1), 164-180.

https://doi.org/10.1111/j.1745-6916.2006.00011.x

Blanco, Á. (2010). Creencias de autoeficacia de estudiantes universitarios: un estudio empírico sobre la especificidad del constructo. Relieve, 1(16), 1-28.

https://doi.org/10.7203/relieve.16.1.4149

Bleicher, R. E. (2004). Revisiting the STEBI-B: Measuring self-efficacy in preservice elementary teachers. School Science and Mathematics, 104(8), 383-391.

Cetinkaya, B. \& Erbas, A. K. (2011). Psychometric properties of the Turkish adaptation of the Mathematics Teacher Efficacy Belief Instrument for in-service teachers. The Spanish Journal of Psychology, 2(14), 956-966. https://doi.org/10.5209/rev_sjop.2011.v14.n2.41

Cid, P., Orellana, A., y Barriga, O. (2010). Validación de la escala de autoeficacia general en Chile. Revista Médica de Chile, 5(138), 551-557. https://doi.org/10.4067/s0034-98872010000500004

Corcoran, K. J. (1995). Understanding cognition, choice, and behavior. Journal of Behavior Therapy and Experimental Psychiatry, 3(26), 201-207. https://doi.org/10.1016/0005-7916(95)00020-z

Cupani, M. (2010). Validity evidence for the new scales for mathematics outcome expectancies and performance goals. Interdiciplinaria, 27, 111-127.

Cupani, M., y Gnavi, G. A. (2007). Un modelo social-cognitivo del rendimiento en Matemática: Estudios de tres. Perspectivas en Psicología, 1(4), 19-27.

Czerniak, C. M. (1990). A study of self-efficacy, anxiety, and science knowledge in preservice elementary teachers. Paper presented at the National Association for Research in Science Teaching, Atlanta, GA.

Czerniak, C. M. \& Lumpe, A. T. (1996). Relationship between teacher beliefs and science education reform. Journal of Science Teacher Education, 4(7), 247-266.

https://doi.org/10.1007/bf00058659 
174 VALIDACIÓN DE LA ESCALA DE CREENCIAS DE EFICACIA EN LA ENSEÑANZA DE LA MATEMÁTICA (ECEEM) Y CARACTERIZACIÓN DE LAS CREENCIAS DE ESTUDIANTES DE PEDAGOGÍA BÁSICA - M. Verdugo, R. Asún y S. Martínez

De Mesquita, P. B. \& Drake, J. C. (1994). Educational reform and self.efficacy beliefs of teachers implementing nongraded primary school programs. Teaching and Teacher Education, 3(10). 291-302. https://doi.org/10.1016/0742-051x(95)97311-9

Dellinger, A. B., Bobbett, J. J., Olivier, D. F., \& Ellett, C. D. (2008). Measuring teachers' self-efficacy beliefs: Development and use of the TEBS-Self. Teaching and Teacher Education, 3(24), 751-766. https://doi.org/10.1016/j.tate.2007.02.010

Dembo, M. H. \& Gibson, S. (1985). Teachers' sense of efficacy: An important factor in school improvement. The Elementary School Journal, 2(86), 173-184. https://doi.org/10.1086/461441

Elosua, P. y Zumbo, B. (2008). Coeficientes de fiabilidad para escalas de respuesta categórica ordenada. Psicothema, 4(20), 896-901.

Enochs, L. G. \& Riggs, I. M. (1990). Further development of an elementary science teaching efficacy belief instrument: A preservice elementary scale. School Science and Mathematics, 8(90), 695-706. https://doi.org/10.1111/j.1949-8594.1990.tb12048.x

Enochs, L., Smith, P., \& Huinker, D. (2000). Establishing factorial validity of the Mathematics Teaching Efficacy Beliefs Instrument. School Science y Mathematics, 4(100), 194-202. https://doi.org/10.1111/j.1949-8594.2000.tb17256.x

Flora, D. B. \& Curran, P. J. (2004). An empirical evaluation of alternative methods of estimation for confirmatory factor analysis with ordinal data. Psychological Methods, 4(9), 466-491.

https://doi.org/10.1037/1082-989x.9.4.466

Forero, C. G., Maydeu-Olivares, A., \& Gallardo-Pujol, D. (2009). Factor analysis with ordinal indicators: A Monte Carlo study comparing DWLS and ULS estimation. Structural Equation Modeling: a Multidisciplinary Journal, 4(16), 625-641.

https://doi.org/10.1080/10705510903203573

Fouad, N. A., Smith, P. L., \& Enochs, L. (1997). Reliability and validity evidence for the middle school self-efficacy scale. Measurement and Evaluation in Counseling and Development, 1(30), 17-30.

Ghaith, G. \& Yaghi, M. (1997). Relationships among experience, teacher efficacy and attitudes toward the implementation of instructional innovation. Teaching and Teacher Education, 4(13), 451-458. https://doi.org/10.1016/s0742-051x(96)00045-5 
Gibson, S. \& Dembo, M. H. (1984). Teacher efficacy: A construct validation. Journal of Educational Psychology, 4(76), 569-582. https://doi.org/10.1037//0022-0663.76.4.569

Gómez, (1997). La alfabetización emocional en educación matemática. Revista de Didáctica de las Matemáticas, 1(13), 7-22.

Guskey, T. R. \& Passaro, P. D. (1994). Teacher efficacy: A study of construct dimensions. American Educational Research Journal, 3(31), 627-643. https://doi.org/10.2307/1163230

Hidalgo, S., Maroto, A., Ortega, T., y Palacios, A. (2014). Influencia del dominio afectivo en el aprendizaje de las matemáticas. En V. Mellado, L. Blanco, A. Borrachero y J. Cárdenas, J. (Ed.), Las emociones en la enseñanza y el aprendizaje de las ciencias y las matemáticas (pp. 218238). Extremadura: Grupo de Investigación DEPROFE.

Hidalgo, S., Maroto, A., y Palacios, A. (2000). Simpatía hacia las matemáticas, las aptitudes y el rendimiento de los alumnos: un complicado triángulo. En actas del IV Simposio de Formación Inicial del Profesorado, Oviedo, España.

Hidalgo, S., Maroto, A., y Palacios, A. (2004). ¿Por qué se rechazan las matemáticas? Análisis evolutivo y multivariante de actitudes relevantes hacia las matemáticas. Revista de Educación, 1(334), 75-95.

Hoffman, B. (2010). "I think I can, but I'm afraid to try": The role of selfefficacy beliefs and mathematics anxiety in mathematics problemsolving efficiency. Learning and Individual Differences, 3(20), 276-283. https://doi.org/10.1016/j.lindif.2010.02.001

Holgado-Tello, F. P., Chacón-Moscoso, S., Barbero-García, I., \& Vila-Abad, E. (2010). Polychoric versus Pearson correlations in exploratory and confirmatory factor analysis of ordinal variables. Quality \& Quantity, 1(44), 153-166. https://doi.org/10.1007/s11135-008-9190-y

Hu, L. T. \& Bentler, P. M. (1999). Cutoff criteria for fit indexes in covariance structure analysis: Conventional criteria versus new alternatives. Structural Equation Modeling, 1(6), 1-55. https://doi.org/10.1080/10705519909540118

Kirsch, I. (1995). Self-efficacy and outcome expectancy: A concluding commentary. En J. E. Maddux (Ed.), Self-efficacy, adaptation, and adjustment: Theory, research, and application (pp. 341-345). New York: Plenum.

Kline, R. B. (2005). Principles and practice of structural equation modeling (2nd Edition ed.). New York: The Guilford Press. 
176 VALIDACIÓN DE LA ESCALA DE CREENCIAS DE EFICACIA EN LA ENSEÑANZA DE LA MATEMÁTICA (ECEEM) Y CARACTERIZACIÓN DE LAS CREENCIAS DE ESTUDIANTES DE PEDAGOGÍA BÁSICA - M. Verdugo, R. Asún y S. Martínez

Ministerio de Educación de Chile, Mineduc. (2015). Estadísticas por carrera. Recuperado de http://www.mifuturo.cl/index.php/futuro-laboral/buscador-por-carre ra?tecnico $=$ false $\&$ cmbareas $=7 \&$ cmbinstituciones $=3$

Ministerio de Educación de Chile, Mineduc. (2016). Currículum en línea. Matemática. Recuperado de http://www.curriculumenlineamineduc.cl/605/w3-propertyvalue-53688. $\mathrm{html}$

Moreira, P. A., Pinheiro, A., Gomes, P., Cotter, M. J., \& Ferreira, R. (2013). Development and evaluation of psychometric properties of an inventory of teachers' perceptions on socio-emotional needs. Psicologia: Reflexão e Crítica, 1(26), 67-76.

https://doi.org/10.1590/s0102-79722013000100008

Moseley, C. \& Utley, J. (2006). The effect of an integrated science and mathematics content-based course on science and mathematics teaching efficacy of preservice elementary teachers. Journal of Elementary Science Education, 2(18), 1-12.

https://doi.org/10.1007/bf03174684

Olaz, F. (2001). La teoría social cognitiva de la autoeficacia. Contribuciones a la explicación del comportamiento vocacional. (Tesis de grado Universidad Nacional de Córdoba, Córdoba, Argentina).

Pajares, F. (1996). Self-efficacy beliefs in academic settings. Review of educational research, 4(66), 543-578.

https://doi.org/10.2307/1170653

Rhodes, R. E. \& Courneya, K. S. (2003). Self-efficacy, controllability and intention in the theory of planned behavior: Measurement redundancy or causal independence? Psychology and Health, 1(18), 79-91. https://doi.org/10.1080/0887044031000080665

Rose, J. S. \& Medway, F. J. (1981). Measurement of teachers' beliefs in their control over student outcome. The Journal of Educational Research, 3(74), 185-190.

https://doi.org/10.1080/00220671.1981.10885308

Rotter, J. B. (1966). Generalized expectancies for internal versus external control of reinforcement. Psychological monographs: General and Applied, 1(80), 1-27.

https://doi.org/10.1037/h0092976

Steiger, J. H. (2007). Understanding the limitations of global fit assessment in structural equation modeling. Personality and Individual Differences, 5(42), 893-898.

https://doi.org/10.1016/j.paid.2006.09.017 
Swars, S. L. (2004). Mathematics teaching efficacy beliefs of elementary preservice teachers and their relationship to mathematics anxiety. (Unpublished doctoral dissertation, University of Alabama, Tuscaloosa, AL, USA).

Swars, S. L. (2005). Examining perceptions of mathematics teaching effectiveness among elementary preservice teachers with differing levels of mathematics teacher efficacy. Journal of Instructional Psychology, 2(32), 139-147.

Tschannen-Moran, M. \& Hoy, A. W. (2001). Teacher efficacy: Capturing an elusive construct. Teaching and teacher education, 7(17), 783-805. https://doi.org/10.1016/s0742-051x(01)00036-1

Usher, E. L. \& Pajares, F. (2009). Sources of self-efficacy in mathematics: A validation study. Contemporary Educational Psychology, 1(34), 89-101. https://doi.org/10.1016/j.cedpsych.2008.09.002

Velásquez, A. (2012). Revisión histórico-conceptual del concepto de autoeficacia. Revista Pequén, 1(2), 148-160.

Wenta, R. G. (2000). Efficacy of preservice elementary mathematics teachers (Unpublished doctoral dissertation, Indiana University, Bloomington, IN, USA).

Williams, D. M. (2010). Outcome expectancy and self-efficacy: Theoretical implications of an unresolved contradiction. Personality and Social Psychology Review, 4(14), 417-425.

https://doi.org/10.1177/1088868310368802

Wirth, R. J. \& Edwards, M. C. (2007). Item factor analysis: Current approaches and future directions. Psychological Methods, 1(12), 58-79. https://doi.org/10.1037/1082-989x.12.1.58

Zalazar, M. F., Aparicio, M. D., Ramírez, C. M., y Garrido, S. J. (2011). Estudios preliminares de adaptación de la Escala de Fuentes de Autoeficacia para Matemáticas. Revista Argentina de Ciencias del Comportamiento, 3(2), 1-6.

Recibido: 25/07/2016

Aceptado: 08/08/2017 


\section{Anexo: ECEEM}

Tabla Al

ECEEM

\begin{tabular}{|c|c|c|c|}
\hline $\begin{array}{l}\mathrm{N}^{\circ} \\
\text { Ittem }\end{array}$ & Frase & Subescala & Dirección \\
\hline Il & $\begin{array}{l}\text { Cuando un estudiante tiene mejores resultados a lo habitual en } \\
\text { matemática, a menudo se debe a que el profesor hizo un esfuerzo } \\
\text { adicional. }\end{array}$ & EREM & + \\
\hline $\mathrm{I} 2$ & $\begin{array}{l}\text { Continuamente voy a encontrar mejores formas para enseñar } \\
\text { matemática. }\end{array}$ & AEM & + \\
\hline I3 & $\begin{array}{l}\text { Aun cuando haga mi mejor esfuerzo, NO enseñaré matemática } \\
\text { tan bien como lo haré en otros subsectores. }\end{array}$ & AEM & - \\
\hline $\mathrm{I} 4$ & $\begin{array}{l}\text { Cuando el rendimiento de los estudiantes mejora, a menudo es } \\
\text { porque el profesor ha encontrado una manera más efectiva de } \\
\text { enseñar. }\end{array}$ & EREM & + \\
\hline $\mathrm{I} 5$ & Sé cómo enseñar conceptos matemáticos de forma efectiva. & AEM & + \\
\hline I6 & NO seré muy efectivo para monitorear actividades matemáticas. & AEM & - \\
\hline I7 & $\begin{array}{l}\text { Si los estudiantes tienen bajo rendimiento en matemática, lo más } \\
\text { probable es que se deba a una enseñanza de la matemática POCO } \\
\text { efectiva. }\end{array}$ & EREM & + \\
\hline 18 & Generalmente enseñaré matemática de forma POCO efectiva. & AEM & - \\
\hline I9 & $\begin{array}{l}\text { La inadecuada formación matemática de un estudiante puede ser } \\
\text { superada con una buena enseñanza. }\end{array}$ & EREM & + \\
\hline $\mathrm{I10}$ & $\begin{array}{l}\text { Cuando un niño o niña con bajo rendimiento progresa en } \\
\text { matemática, es por lo general debido a la atención adicional dada } \\
\text { por el profesor. }\end{array}$ & EREM & + \\
\hline Ill & $\begin{array}{l}\text { Comprendo conceptos matemáticos lo suficientemente bien como } \\
\text { para ser efectivo al enseñar matemática elemental. }\end{array}$ & AEM & + \\
\hline $\mathrm{I} 12$ & $\begin{array}{l}\text { El profesor es generalmente responsable de los logros de los } \\
\text { estudiantes en matemática. }\end{array}$ & EREM & + \\
\hline $\mathrm{I} 13$ & $\begin{array}{l}\text { El logro de los estudiantes en matemática está directamente } \\
\text { relacionado con la eficacia de su profesor al enseñar matemática. }\end{array}$ & EREM & + \\
\hline $\mathrm{I} 14$ & $\begin{array}{l}\text { Si los padres comentan que su hijo o hija muestra mayor interés } \\
\text { en matemática, es probablemente debido al desempeño del } \\
\text { profesor. }\end{array}$ & EREM & + \\
\hline I15 & $\begin{array}{l}\text { Me resultará difícil usar material concreto para explicar a los } \\
\text { estudiantes los por qué de la matemática }\end{array}$ & AEM & - \\
\hline I16 & $\begin{array}{l}\text { Usualmente, seré capaz de responder preguntas matemáticas que } \\
\text { hagan los estudiantes. }\end{array}$ & AEM & + \\
\hline $\mathrm{I} 17$ & $\begin{array}{l}\text { Me pregunto si tendré las habilidades necesarias para enseñar } \\
\text { matemática. }\end{array}$ & AEM & - \\
\hline I18 & $\begin{array}{l}\text { Si tuviera la opción, preferiría NO invitar a un directivo de mi } \\
\text { establecimiento a evaluar mi desempeño enseñando matemática. }\end{array}$ & AEM & - \\
\hline I19 & $\begin{array}{l}\text { En general, yo NO sabría cómo ayudar a un estudiante que tenga } \\
\text { dificultades para entender un concepto matemático. }\end{array}$ & AEM & - \\
\hline $\mathrm{I} 20$ & $\begin{array}{l}\text { Cuando yo enseñe matemática, acogeré las preguntas de los } \\
\text { estudiantes. }\end{array}$ & AEM & + \\
\hline I21 & NO SÉ qué hacer para incentivar a los estudiantes en matemática & AEM & - \\
\hline
\end{tabular}

Fuente: Elaboración propia. 